\title{
Induced hypotension with epidural/general anes- thesia reduces transfusion in radical prostate surgery
}

\author{
[L'hypotension contrôlée, pendant l'anesthésie péridurale et générale combinée
}

pour la prostatectomie radicale, réduit la nécessité de transfusion]

Paul J. O’Connor MB BCh FFARCSI, * John Hanson MSc, $†$ Brendan T. Finucane Md FrCPC FRCA*

Purpose: Radical prostatectomy is associated with substantial blood loss frequently requiring allogeneic blood transfusion. We investigated the efficacy of deliberate hypotension using combined epidural/general anesthesia in reducing allogeneic transfusion requirements in patients undergoing radical prostatectomy.

Methods: In a prospective, randomized, single-blind trial, 102 patients undergoing radical prostatectomy were allocated to either an epidural group $(n=5 \mathrm{I})$ or a control group $(n=5 \mathrm{I})$. In the epidural group, deliberate hypotension was achieved with a target mean arterial pressure of $55-60 \mathrm{mmHg}$. The trigger for allogeneic blood transfusion in both groups was a hematocrit value $<0.25$

Results: Operative blood loss in the epidural group was significantly less than that in the control group $(955 \pm 517 \mathrm{~mL}$ vs 1477 $\pm 823 \mathrm{~mL}$ respectively, $P<0.00 \mathrm{I})$. The percentage of patients who reached the threshold trigger for allogenic transfusion was significantly less in the epidural group ( $8 \%$ vs $26 \%$, respectively, $P=0.019)$ and the number of patients who were actually transfused during hospitalization was also significantly less $(P=$ 0.028). There were no serious adverse events in either group during the study.

Conclusion: Controlled hypotension using a combined epidu$\mathrm{ral} /$ general anesthetic technique is associated with significantly less blood loss, and a reduction in the use of allogeneic blood in patients undergoing radical prostatectomy compared to general anesthesia alone.
Objectif: La prostatectomie radicale est associée à une perte sanguine importante qui nécessite des transfusions allogéniques. Nous avons vérifié l'efficacité d'une hypotension contrôlée pendant l'anesthésie péridurale et générale combinée pour réduire la nécessité de transfusion allogénique chez les patients qui subissent une prostatectomie radicale.

Méthode : Dans une étude prospective, randomisée et à simple insu, 102 patients devant subir une prostatectomie radicale ont été dirigés vers le groupe de péridurale $(n=5 I)$ ou le groupe témoin $(n=5 \mathrm{l})$. Avec la péridurale, une hypotension contrôlée $a$ été réalisée avec une tension artérielle moyenne cible de 55-60 $\mathrm{mmHg}$. Un hématocrite $<0,25$ était le seuil pour l'administration d'une transfusion sanguine allogénique.

Résultats : La perte sanguine opératoire a été significativement moindre dans le groupe de péridurale que dans le groupe témoin $(955 \pm 517 \mathrm{~mL}$ vs $1477 \pm 823 \mathrm{~mL}, P<0,001)$. Le pourcentage de patients chez qui l'hématocrite seuil a déclenché la transfusion $a$ été significativement moindre avec la péridurale (8\% vs $26 \%$, respectivement, $P=0,019$ ) et le nombre de patients transfusés pendant l'hospitalisation a aussi été significativement moindre $(P=0,028)$. Aucun événement indésirable grave n'a été relevé pendant l'étude.

Conclusion: L'hypotension contrôlée pendant une anesthésie péridurale et générale combinée, comparée à l'anesthésie générale seule, est associée à une perte sanguine significativement plus faible et à une réduction de transfusion allogénique chez des patients qui subissent une prostatectomie radicale.

From the Department of Anesthesiology and Pain Medicine, ${ }^{*}$ University of Alberta; and the Division of Health, Population and Information, $\dagger$ Cross Cancer Institute, Edmonton, Alberta, Canada.

Address correspondence to: Dr. Brendan T. Finucane, Department of Anesthesiology and Pain Medicine, University of Alberta, Clinical Sciences Building, Room 8-120, Edmonton, Alberta T6G 2B7, Canada. Phone: 780-407-2876; Fax: 780-407-7461.

E-mail: bfinucan@ualberta.ca

Accepted for publication May 3, 2005

Revision accepted April 3, 2006.

Final revision accepted May 1, 2006.

Competing interests: None declared. 
B LOOD conservation measures are frequently employed in procedures such as radical retropubic prostatectomy (RP) to minimize exposure to allogeneic blood. A recent report cited allogeneic transfusion rates of $15 \%$ amongst patients who predonate (PAD) for this procedure and $19 \%$ with the use of acute normovolemic hemodilution $(\mathrm{ANH})$ without preoperative erythropoietin. ${ }^{1}$

Operative blood loss may be reduced using controlled hypotension, a technique in which mean arterial pressure (MAP) is maintained within a target range of $50-60 \mathrm{mmHg}$ to provide a dry surgical field. ${ }^{2-8} \mathrm{~A}$ previous study of RP reported that $25 \%$ of patients undergoing deliberate hypotension using sodium nitroprusside received allogeneic blood, compared to a transfusion rate of $45 \%$ for $\mathrm{ANH}$ and $60 \%$ for controls. ${ }^{6}$ Epidural anesthesia ${ }^{5}$ may offer some advantage over other techniques for deliberate hypotension, as there is evidence that central neuraxial blockade per $s e$ is associated with fewer blood transfusions in major surgery. ${ }^{9}$ Epidural anesthesia with controlled hypotension has been used in the elderly, ${ }^{10}$ in patients with coexisting hypertension ${ }^{11}$ and is associated with minimal blood loss during total hip arthroplasty. ${ }^{12}$

The use of combined epidural/general anesthesia for controlled hypotension has been evaluated in a preliminary report. ${ }^{13}$ The aim of this study was to compare blood loss and transfusion outcomes using this technique vs general anesthesia alone in patients undergoing radical prostatectomy.

\section{Methods}

One hundred and two patients, ASA physical status I-III, with adenocarcinoma of the prostate presenting for $\mathrm{RP}$ and bilateral pelvic lymphadenectomy were enrolled in a prospective, randomized, singleblind study. Institutional Ethics Board approval was obtained and all patients gave written informed consent. Patients with a history of a bleeding diathesis, aortic or mitral stenosis, uncontrolled hypertension (diastolic blood pressure > $110 \mathrm{mmHg}$ ), myocardial infarction within a year preoperatively, previous cerebrovascular accident, transient ischemic attack within six months, or extensive spinal surgery were excluded from the study. Laboratory exclusion criteria included a hematocrit $<0.39$ or serum creatinine $>$ $150 \mu \mathrm{mol} \cdot \mathrm{L}^{-1}$.

Preoperative investigations before surgery included a complete blood count, serum electrolytes, creatinine and electrocardiogram (ECG). All patients received heparin 5,000 units $s c$ for thromboprophylaxis approximately one hour before the scheduled procedure. Based on a computer-generated table of random numbers, patients were block randomized (block size $=10)$ using blinded study envelopes which were opened immediately prior to surgery. In patients assigned to the epidural group, a combined epidural/ general anesthesia technique was used with a target MAP of 55-60 mmHg. Patients who received general anesthesia alone without controlling for MAP served as controls.

All epidurals were performed with appropriate monitoring using a standard midline approach with patients in the sitting position. Fentanyl 50-75 $\mu \mathrm{g}$ iv was used to provide analgesia for epidural insertion. Catheters were placed in the low thoracic or high lumbar region and were tested for intravascular or subarachnoid placement using $3 \mathrm{~mL}$ of lidocaine $1.5 \%$ with epinephrine $5 \mu \mathrm{g} \cdot \mathrm{mL}^{-1}$. Anesthesia was induced with fentanyl and propofol, and rocuronium was used to facilitate endotracheal intubation. Ventilation was controlled artificially with a mixture of isoflurane 1$2 \%$ and nitrous oxide $60 \%$ in oxygen, to maintain endtidal carbon dioxide in the range of $35-45 \mathrm{mmHg}$. Additional intraoperative monitoring included automated ECG ST-segment analysis, invasive (radial) arterial blood pressure, nasopharyngeal temperature and airway gas analysis. Following induction of anesthesia, a loading dose of ropivacaine $0.5 \%$ was titrated via the epidural catheter until the target MAP was attained. The loading dose was followed by a continuous epidural infusion of ropivacaine $0.2 \%$ plus fentanyl $2 \mu \mathrm{g} \cdot \mathrm{mL}^{-1}$ to maintain MAP within the range of $55-$ $60 \mathrm{mmHg}$. Intravenous morphine sulphate, 10-20 $\mathrm{mg}$, was provided for intraoperative analgesia in the control group. Crystalloids (Ringer's lactate) and colloids (pentaspan) were given according to the usual practice of the attending anesthesiologist. A hematocrit value $<0.25$ as determined by arterial blood gas analysis (ABL 700 series, Radiometer, Copenhagen; calibrated q $4 \mathrm{hr}$ ) was the threshold for allogeneic transfusion in all cases. Values of MAP $<50 \mathrm{mmHg}$ were treated aggressively with iv fluids and vasopressor therapy. Patients who developed unacceptably low blood pressures ( $\mathrm{MAP} \leq 50 \mathrm{mmHg}$ ) were started immediately on an infusion of epinephrine (1-10 $\mu \mathrm{g} \cdot \mathrm{min}^{-1}$ ). An iv fluid warming device ("Hotline", Level 1 Technologies Inc., Rockland, MA, USA) and convective heating blanket ("Bair Hugger", Augistine Medical Inc., Eden Prairie, MN, USA) were utilized to maintain body temperature $>35.5^{\circ} \mathrm{C}$.

Blood loss was measured by subtracting the dry weight from the wet weight of surgical sponges, subtracting the volume of irrigation fluid used from the total volume of fluid in the suction canisters, and adding the two figures. Other data included operating 
TABLE I Patient characteristics

\begin{tabular}{|c|c|c|}
\hline Variable & Control & Study \\
\hline ASA physical class (I/II/III) & $1 / 44 / 5$ & $0 / 43 / 6$ \\
\hline Age $(y r)$ & $64(48-74)$ & $63(51-76)$ \\
\hline Weight (kg) & $89(13)$ & $86(12)$ \\
\hline Height (m) & $1.76(0.07)$ & $1.76(0.07)$ \\
\hline Estimated blood volume ${ }^{14}(\mathrm{~mL})$ & $5490(564)$ & $5409(561)$ \\
\hline Hematocrit & $0.44(0.03)$ & $0.44(0.03)$ \\
\hline Platelet count $\left(\times 10^{9} \cdot \mathrm{L}^{-1}\right)$ & $223(51)$ & $237(64)$ \\
\hline
\end{tabular}

Values are mean (SD or range) unless otherwise stated. No significant differences were observed.

time (from skin incision until last suture) and hospital length of stay (LOS). Investigations on postoperative day one (POD\#1) included hematocrit, serum creatinine and a 12-lead ECG in all cases. Hematocrit measurements were repeated every two days and on the day of discharge, or more frequently if required, and other investigations were performed according to clinical judgment. Outcome surveillance consisted of daily follow-up by study investigators in addition to the usual postoperative care by staff urologists. Serious adverse events (SAEs) were defined as death, symptomatic Q-wave myocardial infarction, stroke, transient ischemic attack, renal impairment (> 20\% increase in serum creatinine that persisted for $>48$ hr), deep-venous thrombosis and pulmonary embolus. Intraoperative ST segment changes were recorded every $15 \mathrm{~min}$. An ischemic episode was defined as a single ST segment deviation from baseline $\leq 0.2 \mathrm{mV}$.

The primary outcome measure was the percentage of patients transfused with allogeneic blood during their entire admission. Data from an audit of all patients undergoing RP at this hospital between 1996 and 1998 indicated the frequency to be $25 \%$. A power analysis based on this figure indicated that a sample size of 49 patients per group would be required to demonstrate an $80 \%$ relative reduction in allogeneic transfusion rates in study patients with a power of $80 \%$ at a $5 \%$ level of significance. ${ }^{14}$ Secondary outcome measures included intraoperative blood loss, operating time, hospital LOS and the occurrence of SAEs. Statistical analysis of the data included Student's t test and Chi-squared test as appropriate. A $P$ value $<0.05$ was considered statistically significant.

\section{Results}

Of 122 patients screened, 14 were excluded, six declined participation, and 102 were randomized. Ninety-nine patients completed the study protocol (one control patient with chronic renal failure was

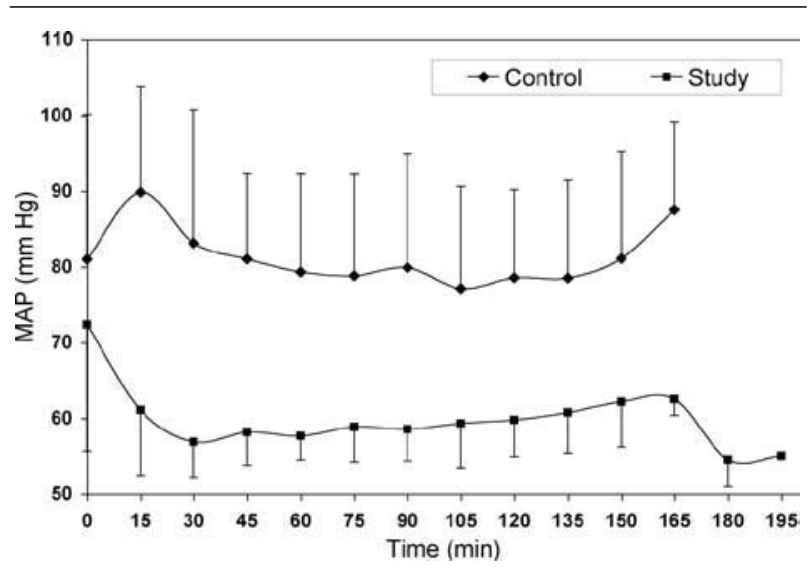

FIGURE 1 Intraoperative mean arterial pressure.

Differences are significant $(\mathrm{p}<0.001)$ except at $\mathrm{t}=0(P=$ $0.20), 150(P=0.039)$ and $165(P=0.046) \mathrm{min} . \mathrm{MAP}=$ mean arterial pressure.

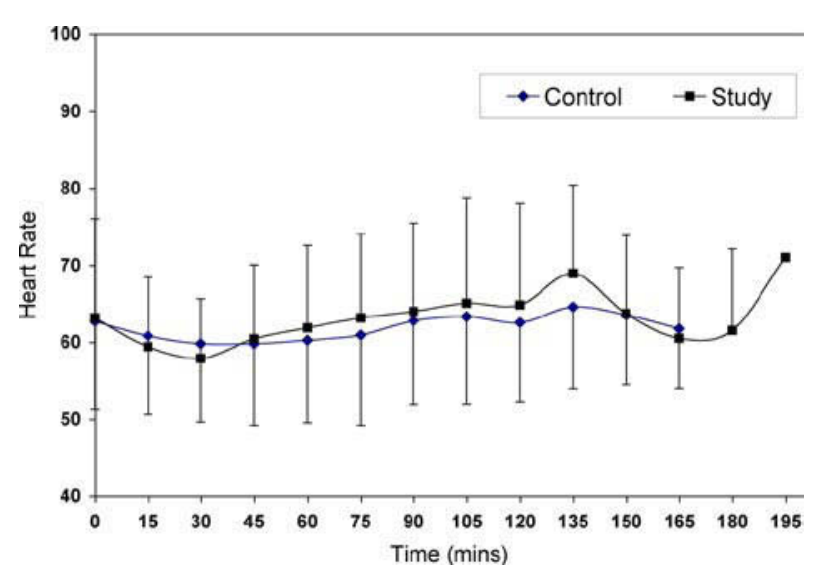

FIGURE 2 Intraoperative heart rates. Values represent means $\pm S D$. There were no signficiant between-groups differences.

enrolled in error and two study patients were withdrawn because of failure to site an epidural catheter).

Patient characteristics were similar in both groups and there was no difference with respect to estimated blood volume, ${ }^{15}$ baseline hematocrit level or platelet counts (Table I). Eighty-two percent of intraoperative MAP values in the study group were within the target range $\pm 5 \mathrm{mmHg}$ (Figure 1 ). The mean heart rates did not vary significantly from the control values (Figure 2 ). Serial changes in mean hematocrit values are shown in Figure 3 and the distribution of minimum hematocrits are displayed in Figure 4. 


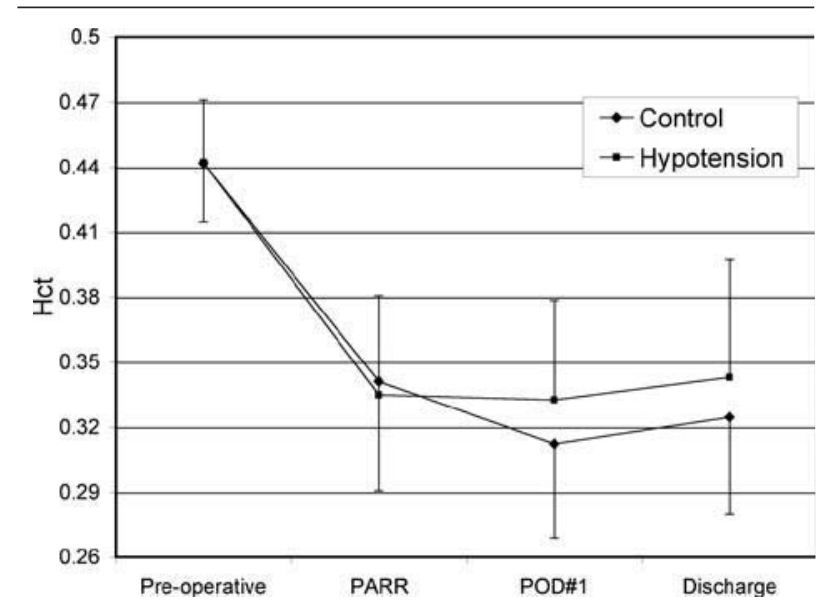

FIGURE 3 Serial changes in hematocrit values. No significant between group differences were observed.

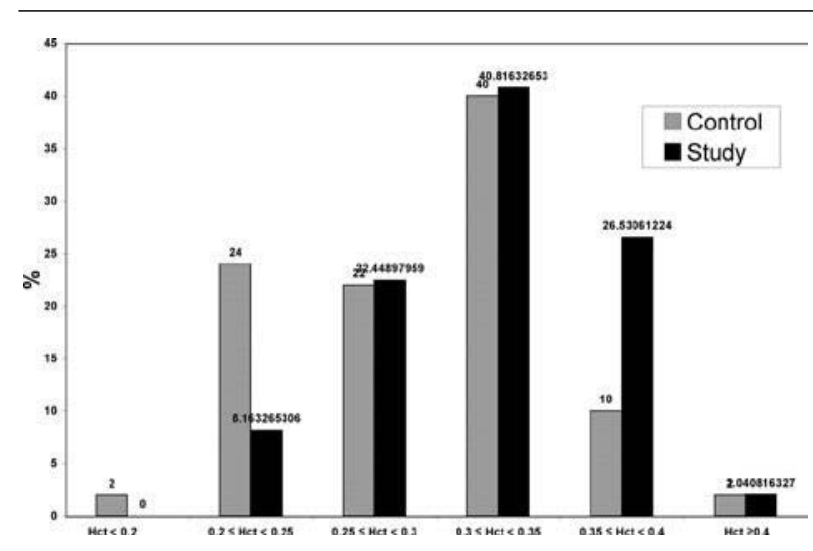

FIGURE 4 Distribution of minimum hematocrits.

Hypotensive anesthesia was associated with a significant reduction in mean blood loss and the proportion of patients meeting transfusion threshold. Hypotensive anesthesia also reduced the proportion of patients requiring allogeneic blood transfusions. The mean volume of crystalloid infused in the epidural group was significantly lower than in the control group, and there was no difference in the volume of colloid infused. Mean operating time in the epidural group was significantly shorter, and there was a trend towards reduced hospital LOS in that group. Complete details of the results including statistical information are presented in Table II.
TABLE II Results

\begin{tabular}{llll}
\hline Parameter & Control & Epidural & $P$ \\
\hline Intraoperative blood loss (mL) & $1477(823)$ & $955(517)$ & 0.001 \\
Number triggered (\%) & $13 / 50(26)$ & $4 / 49(8)$ & 0.019 \\
Number transfused (\%) & $9 / 50(18)$ & $2 / 49(4)$ & 0.028 \\
Intraoperative crystalloid (mL) & $3868(994)$ & $3461(766)$ & 0.025 \\
Intraoperative colloid (mL) & $469(780)$ & $419(429)$ & 0.70 \\
Operating time (min) & $122(32)$ & $107(36)$ & 0.038 \\
Length of stay in days $(>5)$ & $34 / 60(68 \%)$ & $24 / 49(49 \%)$ & 0.055 \\
\hline Values are mean (SD) unless otherwise stated.
\end{tabular}

Nine of 50 patients $(18 \%)$ in the control group received a total of 24 units of packed red blood cells compared with two of 49 patients (4.1\%) who received a total of three units. The median number of units transfused (range) in control patients was 0 (0-4) compared with $0(0-2)$ in study patients. Hematocrit values were routinely measured in the preoperative period, every hour intraoperatively, once in the postanesthesia care unit, and every second day up to and including the day of discharge. The number of patients requiring low doses of epinephrine intraoperatively was $8 / 49(16 \%)$. The median dose of local anesthetic administered intraoperatively was 67 $\mathrm{mg}$ (range 30-125 mg). Patients in the control group received ephedrine or neosynephrine at the discretion of the anesthesiologist when hypotension occurred. Vasopressors were not administered during the postoperative period to patients in either group.

One episode of ST segment depression was recorded in a study patient compared with none in the control group $(P=\mathrm{NS})$. Widespread ST segment depression was noted on the immediate postoperative 12-lead ECG, but the ECG returned to baseline on the first day postoperatively. The CKMB and cardiac troponin values remained within normal values. One patient in the epidural group developed a spinal headache secondary to a dural tap. This resolved with conservative treatment and there were no SAEs during the study.

\section{Discussion}

Clinical concerns regarding the potential adverse effects of allogeneic transfusion as well as logistical problems such as increasing costs and intermittent shortages of blood products, underline the importance of blood conservation. ${ }^{16}$ The risk of transfusion-associated viral illness is extremely low in modern times. ${ }^{17}$ However there is renewed concern about transmission of viral diseases through the blood supply in North America with the increasing prevalence of West Nile virus infection in Canada, the United States, Mexico 
and the Caribbean since 1999. ${ }^{18}$ Some new viruses have been identified in the blood of unpaid donors (hepatitis $\mathrm{G}$ and human herpes virus 8 ). ${ }^{19}$ We recently learned that the new variant of Creutzfeldt-Jakob disease may be transmittable through the blood supply. ${ }^{20}$ Despite these new and perceived threats to the blood supply, transfusion of ABO-incompatible blood, resulting from human error still continues to be the major concern today. ${ }^{21}$

Preoperative autologous donation and $\mathrm{ANH}$ are commonly employed to limit exposure to allogeneic blood. However, PAD is both costly and wasteful while, in addition, it increases the likelihood of exposure to stored blood with its attendant risks. A recent study of RP reported allogeneic transfusion rates of $15 \%$ with PAD, $19 \%$ for ANH and 4\% for ANH combined with preoperative erythropoietin, although the cost of the latter was considerable. ${ }^{1}$ Two other studies $^{6,7}$ have reported allogeneic transfusion rates of $25 \%$ and $0 \%$ among patients undergoing deliberate hypotension with sodium nitroprusside. The latter study demonstrated a reduction in blood loss and overall number of units transfused throughout the study in comparison to controls, but failed to show statistical significance in terms of the proportion of patients receiving allogeneic blood.

The current monitoring protocol for radical prostatectomy does not include central venous pressure measurement but direct arterial pressure monitoring is used routinely. There are a number of studies in the literature demonstrating the benefits of epidural anesthesia on operative time, blood loss and length of hospital stay. The majority of these studies are retrospective chart reviews. ${ }^{22-24}$ This was a prospective study, and an appropriate number of patients were recruited to determine the primary outcome.

This is the first study in which a combined epidu$\mathrm{ral} /$ general anesthesia was used explicitly for deliberate hypotension as a blood conservation technique in radical prostatectomy surgery. The primary endpoint of the study was in-hospital transfusion rates based on a single-variable (hematocrit) transfusion trigger. All patients who triggered during surgery in this study were transfused, although some who triggered postoperatively were not transfused because of nonadherence to the study protocol by surgical staff. Both the number of transfusion triggers ( $8 \%$ vs $26 \%)$ and the number of patients transfused (4\% vs $18 \%$ ) were significantly reduced in the epidural group compared with controls. Operative blood loss was also significantly reduced in the epidural group. Although the accuracy of this measure is limited, the result is consistent with differences in hematocrit between groups.
Mean surgical operating time was reduced by $15 \mathrm{~min}$ in hypotensive patients compared with controls. This difference, sufficient for performing an epidural in most patients, may be a result of improved operating conditions in a 'drier' surgical field. A trend towards reduced LOS was observed in favour of hypotensive patients in this study. This did not reach conventional statistical significance, although the study was not powered to detect such a difference if it did exist.

No SAEs were recorded during in-hospital followup in this study. Although this finding is similar to other recent publications of deliberate hypotension, ${ }^{6-8}$ it is clear that a zero numerator (no SAEs in a study population) does not imply zero risk. In this study population the risk of an SAEs was less than 3\%. ${ }^{25}$ There is evidence that nitroprusside-induced hypotension is associated with a significant increase in acute phase proteins. ${ }^{7}$ This finding may indicate transient gut ischemia secondary to hypotension, although a further study of patients undergoing maxillo-facial surgery (with controlled hypotension using isoflurane or esmolol/nitroglycerin), found that gastric intramucosal $\mathrm{pH}$ values remained above 7.37 leading to the conclusion that splanchnic perfusion was not compromised. ${ }^{8}$ Overall, these findings suggest that deliberate hypotension may be applied to patients who are carefully selected, based on appropriate exclusion criteria. Patients undergoing RP have already been screened by their surgeon and are generally quite healthy. This probably accounts for the overall exclusion rate of only $11 \%$ in our patients.

One may question the ethics of subjecting an older group of patients to induced hypotension when one considers the risks of this technique compared to the risks of receiving a blood transfusion. The estimated risk of mortality from an allogeneic blood transfusion is in the range of $0.0001 \%-0.0004 \%$, and the estimated risk of mortality from induced hypotension may vary between $0.02 \%$ and $0.34 \%$. This is a very gross estimate of the risks of mortality in both categories. The estimated risk of mortality from allogeneic blood transfusion does not take into account the risks of recurrence of cancers associated with blood transfusion or the increased risk of infections associated with blood transfusion. One study demonstrated that patients with colon cancer who received a blood transfusion had twice the infection rate of those who were not transfused (12\% vs 25\%). ${ }^{26}$ Patients who receive blood transfusions have one- and ten-year mortality rates of $24 \%$ and $52 \%$ respectively. ${ }^{27}$ Irrespective of these risks, most patients would prefer not to receive blood if at all possible for many reasons, and in view of the uncertainty about the short- and long-term effects 
of blood transfusion, we should make every effort to avoid this intervention.

Estimating the risk associated with induced hypotension can also be challenging William-Russo ${ }^{10} \mathrm{com}^{-}$ pared outcomes using two different MAP targets (45-55 $\mathrm{mmHg}$ and $55-70 \mathrm{mmHg}$ ) for hypotensive epidural anesthesia in high-risk patients undergoing total hip arthroplasty. They concluded that elderly patients with co-morbidities could safely undergo hypotensive epidural anesthesia, and there was no evidence of a greater overall risk in the markedly hypotensive group. We have already suggested that estimating mortality from blood transfusion is an over- simplification because these estimates do not capture the hidden risks of transfusion. Furthermore, if we estimate the risk of hypotensive epidural anesthesia, we find that the mortality is much lower than the estimate for the broad category of "induced hypotension" which includes all methods of inducing hypotension.

Combined epidural/general anesthesia is frequently used in modern anesthesia, and this technique is associated with hypotension, although it is rarely referred to as "induced" hypotension. This technique has particular advantages in pelvic surgery because it greatly reduces not just arterial pressure, but also venous pressure, thereby potentially reducing venous bleeding from the pelvic vessels. Other methods of induced hypotension may have the same advantage, although there are no published studies comparing conventional methods with induced epidural hypotension. Piper et al. ${ }^{7}$ showed that the intraoperative blood loss achieved with nitroprusside induced hypotension was in the same range as that achieved in the current investigation.

Hypotensive epidural anesthesia has recognized advantages over conventional methods. Reflex tachycardia is frequently reported when using pharmacological agents such as nitroprusside, nitroglycerin, and pentolinium to reduce blood pressure. Beta-adrenergic blockade is frequently required when using these vasodilators. ${ }^{28}$ Tachycardia does not occur with hypotensive epidural anesthesia unless large doses of $\beta$-stimulating agents are used to increase blood pressure. Beta-adrenergic blocking drugs depress myocardial contractility and may be problematic in patients with limited cardiac reserve. Minimum alveolar contration ${ }^{29}$ values are greatly reduced when using combined epidural/general anesthesia, thereby preserving myocardial contractility, and facilitating more rapid recovery from anesthesia. Other advantages of reduced inhalation anesthesia include less postoperative nausea and vomiting, and improved pain control when the regional technique is extended into the postoperative period.
One anesthesiologist (P.O.C.) administered the majority of anesthetics in this study. We were unable to achieve this goal in surgery. Five urologists were involved in these cases. The majority of cases were performed by two surgeons and the distribution of control/study patients was even amongst four of the five urologists. The distribution was uneven for the remaining urologist who completed 15 cases, for whom the distribution of control/study patients was $10 / 5$.

Subcutaneous heparin was administered to all patients on the morning of surgery. Ideally, we would have preferred to hold the heparin until completion of surgery. The Care Map for this procedure at the study institution required that heparin be administered on the morning of surgery. Logistically, it would take several months to change such a protocol. The American Society of Regional Anesthesia guidelines on this topic state that there is no contraindication to the use of neuraxial techniques in the presence of $s c$ heparin. However, the guidelines suggest that the risk of bleeding may be reduced by delaying heparin administration until the block has been established.

The principal limitation of this study is the impossibility of blinding the anesthesiologists involved. Although most transfusions (77\%) were initiated postoperatively by non-study personnel, it is possible that this may have biased the results. Similarly, the use of a rigid laboratory-based transfusion trigger could introduce potential bias due to the effects of hemodilution. It is unlikely that these factors had a significant effect on the outcome of the study for several reasons. Firstly, the trigger rate in the control group $(26 \%)$ was almost identical to the $25 \%$ transfusion rate found in the audit which we performed, and is well in agreement with published studies. ${ }^{1,6}$ Secondly, the hematocrit was consistently higher in the epidural group until hospital discharge, which does not support excessive hemodilution in the control group. Thirdly, there is evidence that volume loading with crystalloid results in greater hemodilution in patients who develop hypotension, than in normotensive controls. ${ }^{30}$ Finally, other outcome measures such as blood loss, operating time, and hospital LOS also favoured the hypotensive group, supporting the principal findings of the study.

It is likely that logistical problems with the blood supply, such as intermittent shortages and increasing costs, will be exacerbated in the future by greater demand for blood products as the population ages. ${ }^{31}$ This study demonstrates the efficacy of controlled hypotension combined with the acceptance of a low hematocrit for blood conservation. It may also help to define acceptable lower limits of hematocrit and intra- 
operative arterial pressure in selected patients. The choice of combined epidural/general anesthesia as the pharmocological means of inducing hypotension also permits the use of epidural analgesia following surgery if so desired.

In conclusion, controlled hypotension using a combined epidural/general anesthesia technique, significantly reduces perioperative blood loss and the need for allogeneic blood transfusion following radical prostate surgery, compared to general anesthesia alone.

\section{Acknowledgements}

The authors sincerely thank the anesthesiologists, urologists and nurses at the University of Alberta Hospital for their cooperation whilst carrying out this study, and Marilyn Blake for her patience during the editing process.

\section{References}

1 Monk TG, Goodnough LT, Brecher ME, Colberg JW, Andriole GL, Catalona WJ. A prospective randomized comparison of three blood conservation strategies for radical prostatectomy. Anesthesiology 1999; 91: 24-33.

2 Enderby GE. Guest editorial. Some observations on the practice of deliberate hypotension. Br J Anaesth 1975; 47: 743-4.

3 Leigh JM. The history of controlled hypotension. Br J Anaesth 1975; 47: 745-9.

4 Thompson GE, Miller RD, Stevens WC, Murray WR. Hypotensive anesthesia for total hip arthroplasty: a study of blood loss and organ function (brain, heart, liver, and kidney). Anesthesiology 1978; 48: 91-6.

5 Sharrock NE, Salvati EA. Hypotensive epidural anesthesia for total hip arthroplasty. A review. Acta Orthop Scand 1996; 67: 91-107.

6 Boldt J, Weber A, Mailer K, Papsdorf M, Schuster P. Acute normovolaemic haemodilution vs controlled hypotension for reducing the use of allogeneic blood in patients undergoing radical prostatectomy. $\mathrm{Br} \mathrm{J}$ Anaesth 1999; 82: 170-4.

7 Piper SN, Suttner SW, Schmidt CC, Kumle B, Maleck $W H$, Boldt J. Acute phase response to nitroprussideinduced controlled hypotension in patients undergoing radical prostatectomy. Anaesthesia 2000; 55: 131-6.

8 Andel D, Andel H, Horauf K, Felfernig D, Millesi W, Zimpfer $M$. The influence of deliberate hypotension on splanchnic perfusion balance with use of either isoflurane or esmolol and nitroglycerin. Anesth Analg 2001; 93: 1116-20.

9 Rodgers A, Walker N, Schug S, et al. Reduction of postoperative mortality and morbidity with epidural or spinal anaesthesia: results from overview of randomised trials. BMJ 2000; 321: 1-12.

10 Williams-Russo P, Sharrock NE, Mattis S, et al. Randomized trial of hypotensive epidural anesthesia in older adults. Anesthesiology 1999; 91: 926-35.

11 Sharrock NE, Mineo R, Urqubart B. Haemodynamic effects and outcome analysis of hypotensive extradural anaesthesia in controlled hypertensive patients undergoing total hip arthroplasty. Br J Anaesth 1991; 67: $17-25$.

12 Sharrock NE, Mineo R, Urqubart B, Salvati EA. The effect of two levels of hypotension on intraoperative blood loss during total hip arthroplasty performed under lumbar epidural anesthesia. Anesth Analg 1993; 76: $580-4$.

13 O'Connor PJ, Wollin TA, Finucane BT. Controlled hypotension with combined epidural / general anesthesia reduces blood loss and transfusion requirements compared with general anesthesia alone in radical prostatectomy. Anesth Analg 2001; 92(2S): S337.

14 Campbell MJ, Julious SA, Altman DG. Estimating sample sizes for binary, ordered categorical, and continuous outcomes in two group comparisons. BMJ 1995; 311: 1145-8.

15 Nadler SB, Hidalgo JU, Bloch T. Prediction of blood volume in normal human adults. Surgery 1961; 51: 224-32.

16 Spahn DR, Casutt M. Eliminating blood transfusions. New aspects and perspectives. Anesthesiology 2000; 93: 242-55.

17 Schreiber GB, Busch MP, Kleinman SH, Korelitz JJ. The risk of transfusion-transmitted viral infections. The Retrovirus Epidemiology Donor Study. N Engl J Med 1996; 334: 1685-90.

18 Gould LH, Fikrig E. West Nile virus; a growing concern? J Clin Invest 2004; 113: 1102-7.

19 Cantaloube JF, Gallian P, Biagini P, et al. Prevalence of $\mathrm{GB}$ virus type $\mathrm{C} /$ hepatitis $\mathrm{G}$ virus RNA and antiE2 among blood donors in Southeastern France. Transfusion 1999; 39: 95-102.

20 Turner ML, Ironside JW. New variant CreutzfeldtJakob disease: the risk of transmission by blood transfusion. Blood Rev 1998; 12: 255-68.

21 Krombach J, Kampe S, Gathof BS, Diefenbach C, Kasper $S M$. Human error: the persisting risk of blood transfusion: a report of five cases. Anesth Analg 2002; 94: 154-6.

22 Shir $\Upsilon$, Raja SN, Frank SM Brendler CB. Intraoperative blood loss during radical retropubic prostatectomy: epidural versus general anesthesia. Urology 1995; 45: 993-9.

23 Frank E, Sood OP, Torjman M, Mulholland SG, Gomella $L G$. Postoperative epidural analgesia following radical 
retropubic prostatectomy: outcome assessment. J Surg Oncol 1998; 67: 117-20.

24 Dunet F, Pfister C, Deghmani M, Meunier $\Upsilon$, Demeilliers-Pfister G, Grise P. Clinical results of combined epidural and general anesthesia procedure in radical prostatectomy management. Can J Urol 2004; 11: 2200-4.

25 Hanley JA, Lippman-Hand A. If nothing goes wrong, is everything all right? Interpreting zero numerators. JAMA 1983; 249: 1743-5.

26 Vamvakas EC, Taswell HF. Long term survival after blood transfusion. Transfusion 1994; 34: 471-7.

27 Heiss MM. Risk of allogeneic transfusions. Br J Anaesth 1998; 81(Suppl 1): 16-9.

28 Andel D, Andel H, Horauf K, Felfernig D, Millesi W, Zimpfer $M$. The influence of deliberate hypotension on splanchnic perfusion balance with use of either isoflurane or esmolol and nitroglycerin. Anesth Analg 2001; 93: 1116-20.

29 Hodgson PS, Liu SS, Gras TW. Does epidural anesthesia have general anesthetic effects? A prospective, randomized, double-blind placebo controlled trial. Anesthesiology 1999; 91: 1687-92.

30 Drobin D, Habn RG. Time course of increased haemodilution in hypotension induced by extradural anaesthesia. Br J Anaesth 1996; 77: 223-6.

31 Goodnough LT, Brecher ME, Kanter MH, AuBuchon JP. Transfusion medicine. First of two parts. Blood transfusion. N Engl J Med 1999; 340: 438-47. 